\title{
PERSONALIDADE DAS MÃES E GRAVIDADE DE SINTOMAS DE TRANSTORNO DO ESPECTRO DO AUTISMO NOS FILHOS
}

\author{
MOTHERS' PERSONALITY AND SEVERITY OF AUTISM SPECTRUM \\ DISORDER SYMPTOMS IN CHILDREN
}

\author{
Lara Araújo Roseira Cannone', Ananda Evelin Genonádio da Silva Menezes', \\ Lucas Almeida Santana Rocha², Saulo Leal Merelles ${ }^{3}$, \\ Maria Thereza Ávila Dantas Coelho ${ }^{4}$, Milena Pereira Pondé 5 \\ Autora para correspondência: Lara Araújo Roseira Cannone - laracannone@gmail.com \\ 'Graduanda em Psicologia na Escola Bahiana de Medicina e Saúde Pública. Salvador, Bahia, Brasil. \\ ${ }^{2}$ Graduando em Medicina na Escola Bahiana de Medicina e Saúde Pública. Salvador, Bahia, Brasil. \\ ${ }_{3}^{3}$ ssiquiatra da Infância e Adolescência no Centro Especializado em Reabilitação, Obras Sociais Irmã Dulce. Salvador, Bahia, Brasil. \\ ${ }^{4}$ Psicóloga. Doutora em Saúde Coletiva. Professora na Universidade Federal da Bahia. Salvador, Bahia, Brasil. \\ ${ }^{5}$ Médica. Doutora em Saúde Coletiva. Professora na Escola Bahiana de Medicina e Saúde Pública. Salvador, Bahia, Brasil.
}

RESUMO I Introdução: Estudo prévio indica que a gravidade dos sintomas de TEA nos filhos parece estar positivamente associada com a gravidade dos sintomas de depressão e ansiedade de pais de crianças com TEA. Esse estudo tem como objetivo analisar a associação entre traços de personalidade das mães e gravidade dos sintomas de TEA nos filhos. Metodologia: a amostra do estudo foi selecionada entre pais de crianças diagnosticadas com autismo em instituições de atendimento a pessoas com TEA. As crianças foram diagnosticadas por profissionais da psiquiatria, segundo os critérios do DSM-5. No total foram incluídas 54 mães. $O$ instrumento utilizado para avaliar os traços de personalidade das mães foi o Inventário Fatorial de Personalidade (IFP). Resultados: alguns traços de personalidade se apresentaram de forma diferentes em mães de crianças com TEA Leve em relação a mães de crianças de sintomas de TEA Moderado ou Grave, especificamente intercepção, deferência, desempenho, exibição, autonomia e ordem. Os traços de assistência, afago, afiliação, agressão e mudança apresentaram baixa expressão em ambos os grupos. $O$ traço de persistência foi o que apresentou maior prevalência de escores elevados nos dois grupos. Conclusão: Apesar de ser um estudo preliminar, com amostra pequena e sem grupo de comparação, este estudo pode servir como indicador para pesquisas futuras com populações maiores que possam esclarecer de forma mais confiável a associação entre traços de personalidade das mães e gravidade dos sintomas nos filhos com diagnóstico de TEA.

Palavras-chave: transtorno do espectro do autismo, personalidade, pais.

\begin{abstract}
Introduction: Previous study indicates that the severity of ASD symptoms in children appears to be positively associated with the severity of the symptoms of depression and anxiety in the parents of children with ASD. This study aims to analyze the association between the personality traits of the mothers and the severity of the ASD symptoms in the children. Methodology: the study sample was selected among parents of children diagnosed with autism in institutions for the care of people with ASD. The children were diagnosed by psychiatry professionals according to DSM-5 criteria. In total, 54 mothers were included. The instrument used to evaluate the personality traits of the mothers was the Factorial Personality Inventory (IFP). Results: some personality traits presented differently in mothers of children with mild ASD in relation to mothers of children with symptoms of moderate or severe ASD, specifically: interception, deference, performance, display, autonomy and order. The traits of care, caring, affiliation, aggression and change presented low expression in both groups. The persistence trait was the one with the highest prevalence of high scores in both groups. Conclusion: Despite being a preliminary study, with a small sample and without a comparison group, this study may serve as an indicator for future research with larger populations that can more reliably clarify the association between the personality traits of the mothers and the severity of the symptoms in children diagnosed with ASD.
\end{abstract}

Key words: autism spectrum disorder, personality, parents. 


\section{INTRODUÇÃO}

O Transtorno do Espectro Autista (TEA) é um transtorno do neurodesenvolvimento caracterizado por prejuízo na interação social recíproca, comunicação social e padrões de comportamento estereotipados e repetitivos. O TEA divide-se em três níveis de gravidade, baseado na quantidade de suporte que $\bigcirc$ paciente demande (APA, 2014). Existem características clínicas associadas ao transtorno de base que pioram $\circ$ prognóstico da criança afetada: epilepsia, retardo mental, distúrbios do sono, hiperatividade, auto e heteroagressividade e irritabilidade (Novaes, Pondé \& Freire, 2008; Pondé, Novaes \& Losapio, 2010). A partir da confirmação do diagnóstico, sabe-se que a interferência do quadro transcende 0 sujeito afetado, pois a família e outras pessoas inseridas em sua rede de apoio social também participam dos desafios e dificuldades causados por tal condição.

Os pais de crianças com TEA podem ter uma maior probabilidade de apresentar algum transtorno mental, tanto por razões genéticas quanto ambientais. Como se trata de um transtorno com possível herança genética, os pais podem ter características do transtorno apresentado pelo filho, ainda que sejam traços atenuados. Com relação ao estresse ambiental, os pais experimentam a sobrecarga de cuidar de uma criança que é muito dependente e não desenvolve as habilidades esperadas. Os pais de crianças com TEA podem ter um risco maior de sofrer de transtornos de ansiedade, transtornos de humor e sintomas obsessivos do que os pais de crianças com síndrome de Down (Cook et al., 1994). A prevalência de transtornos de ansiedade ao longo da vida foi significativamente maior em pais de crianças com TEA do que em pais de crianças com Síndrome de Down (Piven et al., 1997). Os traços de ansiedade em pais de crianças com TEA foram relacionados em parte à sobrecarga de cuidar da criança (Murphy et al., 2000). Um estudo de caso-controle foi conduzido para comparar o estado mental de mães de crianças com TEA, mães de crianças com síndrome de Down ou mães de crianças típicas. Houve mais mulheres com risco de transtorno mental no grupo de mães de crianças com TEA, do que nos dois outros grupos (Shu, Lung \& Chang., 2000). Transtornos psiquiátricos foram encontrados em $15,7 \%$ das mães de crianças com TEA, estimativa que foi significativamente maior do que $8,2 \%$ encontrado no grupo controle (Mouridsen, 2007).

Com relação à personalidade de pais de indivíduos com TEA, Murphy et al. (2000) e Piven et al. (1997) sugerem um perfil de maior rigidez, indicado por uma menor capacidade de ajuste a mudanças em pais de indivíduos com TEA, comparando com pais de crianças com Síndrome de Down. Estudo prévio usando o IFP para avaliar o perfil de personalidade sugere que $\circ$ perfil de personalidade de pais de sujeitos com TEA se caracteriza por um perfil de indivíduos que apresentam resistência em mudar seus hábitos ou experimentar coisas novas; predominância de perfis de personalidade com pouco interesse ou prazer de estar com as pessoas; assim como pessoas com baixa capacidade de abstração e imaginação (Rocha, 2015).

A gravidade dos sintomas de TEA parece estar positivamente associada com a gravidade dos sintomas de depressão e ansiedade nos pais de crianças com TEA (Machado, Celestino, Serra, Caron \& Pondé, 2016). Não há estudos discutindo a associação entre traços da personalidade das mães e gravidade dos sintomas dos filhos com TEA. Define-se personalidade como um conjunto de características explícitas e implícitas e a relação dessas características com o meio (Millon \& Davis, 1996). Por se tratar de um construto dialético, no qual traços individuais estão em constante interação com o ambiente, é interessante pensar que alguns traços em comum entre pais e seus filhos com TEA podem ser relevantes para entender possíveis comportamentos das crianças e o seu progresso no tratamento. Esse estudo tem como objetivo analisar a associação entre traços de personalidade das mães e gravidade dos sintomas de TEA nos filhos.

\section{MÉTODOS}

A população alvo foram pacientes atendidos em uma escola especializada para pessoas com TEA, 
pacientes que estavam na lista de espera para atendimento nessa escola e pacientes de consultório privado. Os pais das crianças da escola foram convidados a participar da pesquisa enquanto esperavam os seus filhos no pátio da escola, ou quando eram atendidos por psiquiatra que presta assistência à escola através de convênio com a universidade. Os pais de criança da lista de espera da escola foram convidados por telefone a participar da pesquisa, tendo como retorno a avaliação psiquiátrica dos seus filhos. Os pais do consultório privado foram convidados pelo psiquiatra assistente a participar da pesquisa. Foram excluídos os pais que não aceitaram participar da pesquisa, os iletrados, os pais adotivos e aqueles que responderam aos questionários de forma inadequada. A amostra foi constituída por 54 mães de indivíduos com diagnóstico clínico de TEA em diferentes graus de gravidade, de ambos os sexos/gêneros, entre 1 e 30 anos de idade. $O$ diagnóstico dos filhos foi feito com a avaliação clínica de psiquiatras a partir de critérios preconizados pelo DSM-5. Os dados foram coletados entre abril de 2014 e abril de 2015.

O instrumento utilizado para avaliar traços de personalidade dos pais foi o IFP (Inventário Fatorial de Personalidade). Trata-se de um teste de avaliação de personalidade, sendo adequado para níveis de aplicação grupal e/ou individual e se destina a pessoas com idade mínima de 18 anos e máxima de 60 anos de idade. Seu preenchimento é auto-administrável. $O$ IFP se propõe a avaliar necessidades básicas com base no estudo coordenado por Henry Murray, em 1938, na Clínica de Psicologia de Harvard, sendo o resultado final reconhecido como a teoria denominada de Personologia. O teste é composto de 15 aspectos a serem avaliados sobre a personalidade do indivíduo, cada um dois quais é avaliado através de nove frases, que se encontram dispostas aleatoriamente ao longo dos 135 itens propostos no questionário. As frases têm a possibilidade de respostas variando de de 1 até 7 , sendo 1 quando - item é considerado como 'nada característico' até 7, que é 'totalmente característico', sendo permitida apenas uma resposta por frase. Além dos 135 itens para avaliar os diferentes aspectos da personalidade são propostos 20 itens referentes à validade das respostas. O resultado de cada aspecto é dividido em percentis, de acordo com os escores, sendo escores altos, medianos ou baixos, correspondendo, respectivamente a percentil $\geq 70$, percentil entre 69 e 31 e percentil $\leq 30$.

Um total de 15 sub-escalas definem 15 traços de personalidade: Assistência (Ass); Dominância (Do); Ordem (O); Denegação (De); Intracepção (I); Desempenho (Des); Exibição (Ex); Heterossexualidade (Het); Afago (Afl); Mudança $(M)$; Persistência (Pers); Agressão (Ag); Deferência (Def); Autonomia (Aut) e Afiliação (Af). A Assistência expressa os desejos e sentimentos de piedade, compaixão e ternura, pelos quais o sujeito deseja dar simpatia e gratificar as necessidades de um sujeito indefeso, defendê-lo do perigo, dar-lhe suporte emocional e consolo na tristeza, doença e outros infortúnios. Já o sujeito Intraceptivo se deixa conduzir por sentimentos e inclinações difusas; - sujeito é dominado pela busca da felicidade, pela fantasia e imaginação. Por sua vez, ○ Afago representa a busca de apoio e proteção, sofre de sentimentos de abandono e ansiedade, insegurança e desespero. A Deferência se relaciona com respeito, admiração e reverência. A Afiliação significa dar e receber afeto de amigos, além de confiança, boa vontade e amor. Por Dominância entende-se sentimentos de autoconfiança e o desejo de controlar os outros, influenciar ou dirigir o comportamento deles através de sugestão, sedução, persuasão ou comando. $O$ que caracteriza Denegação é o desejo ou tendência de se submeter passivamente à força externa; aceitar desaforo, castigo e culpa; resignarse ao destino, admitir inferioridade, erro e fracasso. Desempenho trata-se de ambição e empenho, desejo de realizar algo difícil, como dominar, manipular e organizar objetos, pessoas e ideias. $O$ entendimento de Exibição o desejo de impressionar, ser ouvido e visto, a vaidade. A Agressão reflete raiva, a imitação e o ódio, tais pessoas gostam de lutar, brigar, atacar. Já Ordem representa a tendência de manter limpeza, organização, equilíbrio e precisão. Persistência expressa o sentimento de continuar qualquer trabalho iniciado por mais difícil que possa parecer. Mudança é o apreço por novidade, aventura, não temer coisas que fogem da rotina. Autonomia é a independência, agir livremente por seus próprios impulsos. E para finalizar, o fator Heterossexualidade fala do desejo de manter relações, desde românticas até sexuais, com indivíduos do sexo oposto. Esse item 
não foi considerado na análise, pois foi considerado inadequado em estudos de validação, de modo que não consta mais na versão subsequente do IFP (Pasquali, Azevedo \& Ghesti, 1997).

A análise dos dados se deu pelo programa de análise estatística SPSS. As informações coletadas através do Inventário Fatorial de Personalidade foram digitadas no referido programa e posteriormente ao fim das aplicações dos questionários, as análises foram geradas no SPSS. Em função do pequeno tamanho da amostra, os dados foram apresentados em termos percentuais, sem nenhum teste de inferência estatística.

O estudo foi aprovado pelo CEP da Escola BAHIANA de Medicina e Saúde Pública em 12/03/2014 através do parecer 551.381 (CAAE 17985613.1.0000.5544).

\section{RESULTADOS}

Um total de 54 pessoas participaram deste estudo até o fechamento da análise, todas mães de crianças com diagnóstico confirmado de TEA, com média de idade de 36 anos, $54 \%$ com segundo grau completo e $54 \%$ com algum trabalho remunerado. Os sujeitos do estudo foram divididos em dois grupos, o Grupo 1 correspondendo às mães de crianças com autismo leve e o Grupo 2 mães de crianças com autismo moderado ou grave, de acordo com os critérios do DSM-5. Os gráficos apresentados (Gráfico 1, 2 e 3) mostram como cada traço de personalidade caracterizado pelo IFP se distribui nos dois grupos.

Considerando os índices de Desejabilidade Social, que indicam a necessidade do indivíduo em mostrarse de forma socialmente adequada e ser aceito pelo coletivo, $50 \%$ das mães de crianças diagnosticadas como TEA leve (Grupo 1) e 78,3\% das mães de crianças diagnosticadas com TEA moderado ou grave (Grupo 2) apresentaram escore alto ( $\geq 70$ ).
Quanto à Validade, o Grupo 2 correspondeu em absoluto aos critérios positivos, o Grupo 1 teve desvio de apenas $9,1 \%$.

Com relação aos traços psicológicos, estimou-se escores baixos ( $\leq 30$ ) em Assistência para 45,5\% das mães do Grupo 1 e 52,2\% das mães do Grupo 2. Quanto à necessidade básica de Intracepção, estimou-se escores baixos para $72,7 \%$ das mães do Grupo 1 e 47,8\% para as mães do Grupo 2. Sobre Afago, os escores foram predominantemente baixos ( $\leq 30$ ) para ambos os grupos: $77,3 \%$ das mães do Grupo 1 e 73,9\% das mães do Grupo 2. Em relação à Deferência, nota-se que os escores elevados ( $\geq$ 70) foram mais prevalentes no Grupo 2 das em relação ao Grupo 1 (21,7\% x 9,1\%). Para Afiliação ambos os grupo tiverem predominância de escores baixos ( $\leq 30$ ): Grupo 1 com $54, \%$ e $60,9 \%$ no Grupo 2, sem diferença importante entre os dois grupos. (Gráfico 1).

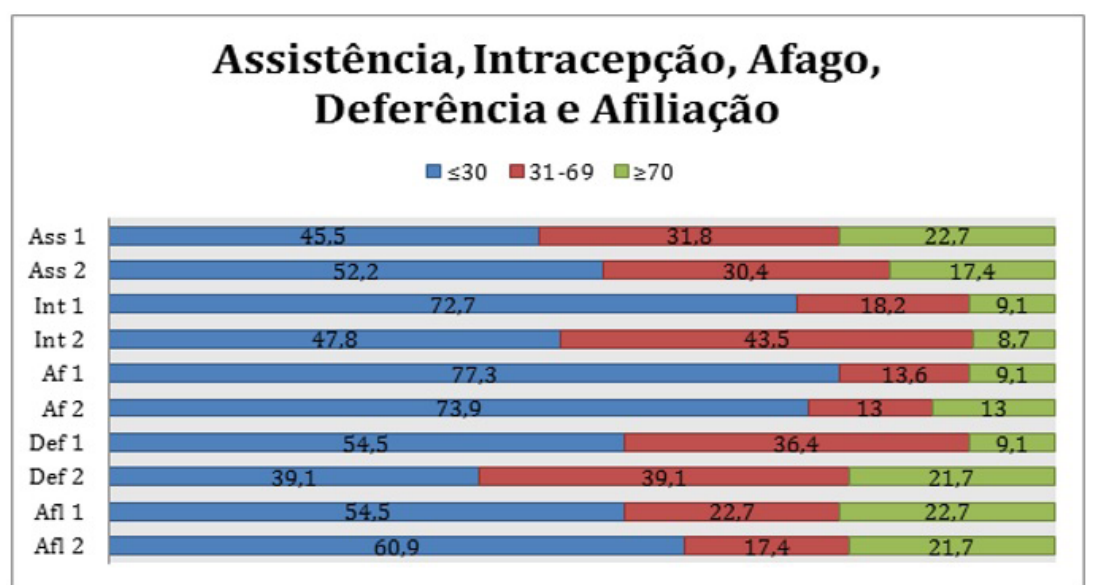

Ass 1 - Assistência nas mães do Grupo 1

Ass 2 - Assistência nas mães do Grupo 2

Int 1 - Intracepção nas mães do Grupo 1 Int 2 - Intracepção nas mães do Grupo 2

Af 1 - Afago nas mães do Grupo 1

Af 2 - Afago nas mães do Grupo 2

Def 1 - Deferência nas mães do Grupo 1

Def 2 - Deferência nas mães do Grupo 2

Af 1 - Afiliação nas mães do Grupo 1

Afl 2 - Afiliação nas mães do Grupo 2

Gráfico 1. Distribuição dos percentis dos Motivos de personalidade Assistência, Intracepção, Afago, Deferência e Afiliação entre as mães do Grupo 1 e 2. 
Expressam-se percentis mais baixos ( $\leq 69$ ) para Dominância em $72,8 \%$ do Grupo 1 e 65,2\% do Grupo 2. A Denegação apresentou-se semelhante em ambos os grupos, porém com predominância de escores $\leq 69: 82 \%$ do Grupo 1 e 78,3\% do Grupo 2. Para Desempenho, os escores altos ( $\geq 70$ ) foram mais prevalentes no Grupo 2 das mães 2 em relação ao Grupo 1 (17,4\% x 9,1\%). Os escores de Exibição foram predominantemente mais baixos ( $\leq 30$ ) em ambos os grupos, porém mais baixos no Grupo 2 em relação ao Grupo 1 (73,9\% x 54,5\%). Os índices de Agressão merecem relevância pela elevada prevalência de escores baixos $(\leq 30)$ em ambos os grupos: $86,4 \%$ no Grupo 1 e e $82,6 \%$ no Grupo 2. (Gráfico 2).

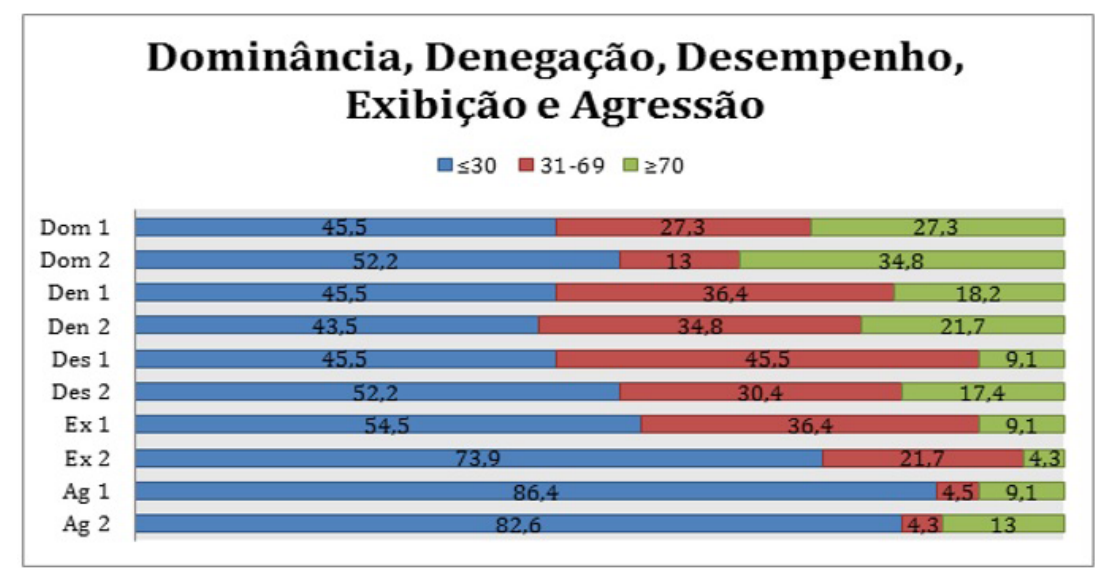

Dom 1 - Dominância nas mães do Grupo 1 Dom 2 - Dominância nas mães do Grupo 2 Den 1 - Denegação nas mães do Grupo 1 Den 2 - Denegação nas mães do Grupo 2 Des 1 - Desempenho nas mães do Grupo 1 Des 2 - Desempenho nas mães do Grupo 2 Ex 1 - Exibição nas mães do Grupo 1 Ex 2 - Exibição nas mães do Grupo 2 Ag 1 - Agressão nas mães do Grupo 1 Ag 2 - Agressão nas mães do Grupo 2

Gráfico 2. Distribuição dos percentis dos Motivos de personalidade Dominância, Denegação, Desempenho, Exibição e Agressão entre as mães do Grupo 1 e 2.

Para o traço Autonomia, observa-se uma prevalência maior de escores $\leq 30$ para as mães do Grupo 2 em relação ao Grupo 1 (52,2\% × 36,4\%). Para os resultados do traço Mudança, chama atenção uma prevalência muito baixa de percentil $\geq 70$ em ambos os grupos: 4,5\% para o Grupo 1 e 4,3\% para o Grupos 2 . Sobre o motivo psicológico Ordem a prevalência de escores mais baixos $(\leq 30)$ foi maior no Grupo $2(69,6 \%)$ em relação ao Grupo 1 (50\%). Já a Persistência foi o motivo que teve maior prevalência de escores mais altos (percentil $\geq 70$ ) em ambos os grupos: $22,7 \%$ no Grupo 1 e 26,1\% no Grupo 2. (Gráfico 3).

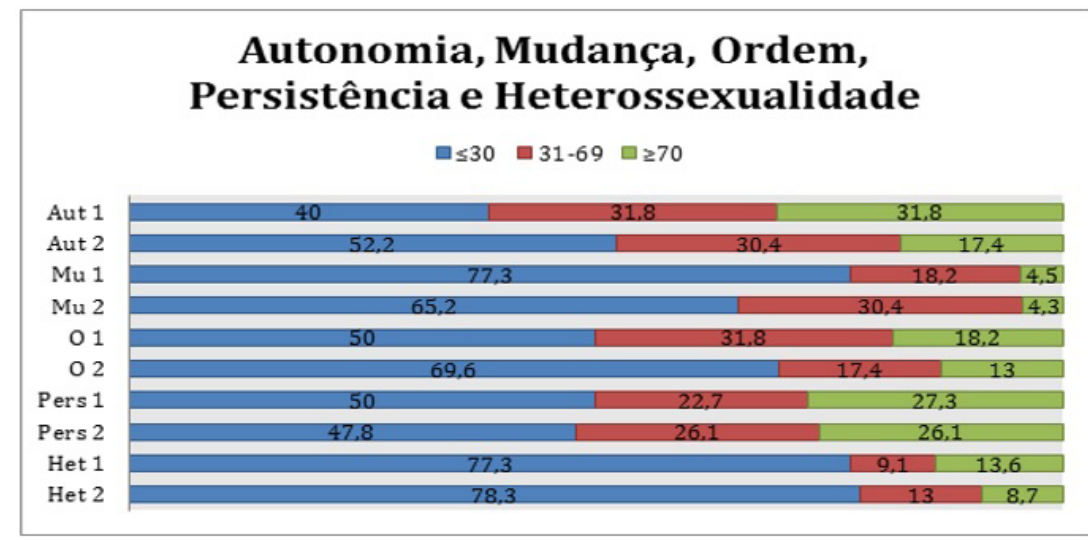

Aut 1 - Autonomia nas mães do Grupo 1 Aut 2 - Autonomia nas mães do Grupo 2 Mu 1 - Mudança nas mães do Grupo 1 Mu 2 - Mudança nas mães do Grupo 2 O 1 - Ordem nas mães do Grupo 1 O 2 - Ordem nas mães do Grupo 2 Pers 1 - Persistência nas mães do Grupo 1 Pers 2 - Persistência nas mães do Grupo 2 Het 1 - Heterossexualidade nas mães do Grupo 1 Het 2 - heterossexualidade nas mães do Grupo 2

Gráfico 3. Distribuição dos percentis dos traços de personalidade Autonomia, Mudança, Ordem, Persistência e Heterossexualidade entre as mães do Grupo 1 e 2.

\section{DISCUSSÃO}

Buscando identificar traços de personalidade que diferenciem $\circ$ grupo de mães de pessoas com TEA moderado ou grave em relação às mães de pessoas com TEA leve, de acordo com a classificação do 
DSM-5, houve sete traços com tendência a indicar diferenças nos dois grupos de mães: Desejabilidade Social, Intracepção, Deferência, Desempenho, Exibição, Autonomia e Ordem. A Desejabilidade Social teve predominância de escores elevados em ambos os grupos, sendo maior entre mães de crianças com sintomas mais graves. Mães de crianças com TEA mais grave apresentam escores mais elevados do traço Deferência do que mães de crianças com TEA leve. Esses dois achados sugerem que mães de crianças com sintomas mais graves podem ter uma maior necessidade de se sentir aceitas pelo coletivo e por pessoas que julgam superiores. Foi previamente relatado uma associação positiva entre sintomas graves de TEA nos filhos e sintomas graves de ansiedade nas mães, porém esses sintomas se mostravam atenuados pela presença do apoio social do pai da criança (Machado et al., 2016), o que está em consonância com os achados do presente estudo, que sugere que as mães de filhos com sintomas mais graves, podem ter uma maior demanda de apoio e aceitação social. Para - traço Desempenho, os escores elevados foram mais prevalentes para as mães com sintomas mais graves. Esse traço reflete a ambição e empenho de realizar algo difícil, podendo, portanto, refletir a situação de maior desafio ambiental que essas mães vivem, em relação àquelas que tem filhos com sintomas mais leves (Machado et al., 2016).

Para o traço Exibição os escores foram predominantemente baixos para ambos os grupos, refletindo a literatura científica que sugere que pais de autistas apresentam déficits sutis em aspectos do comportamento social, semelhante ao que é observado nos indivíduos com TEA (Yucel et al., 2015). Os escores foram mais baixos ainda para as mães de crianças com sintomas mais graves; podendo revelar um maior retraimento deste grupo de mães em relação àquelas que tem sintomas mais leves, indicando assim que o traço de personalidade relacionado a menor sociabilidade apresenta-se com maior intensidade em mães de crianças com sintomas mais graves. As mães de crianças com TEA leve possuem mais Autonomia em relação às mães de crianças com TEA mais grave. É possível que as mães de crianças com sintomas mais leves tenham menos sobrecarga no cuidado aos filhos e, por conseguinte, mais autonomia e liberdade em relação a mães de pessoas com espectro mais grave de TEA, hipótese que pode ser testada em estudos futuros, uma vez que não encontramos na literatura nenhuma referência a esse achado.

Para os demais traços não houve diferença aparente entre os dois grupos. Para ambos os grupos houve predominância de escores baixos para os traços Assistência, Afago, Afiliação, Agressão e Mudança. A baixa expressão do traço Assistência, que diz respeito a empatia, relacionada a sentimentos de piedade, compaixão e ternura, pode ser explicada como traço do espectro do autismo presente de forma atenuada nas mães, o que está de acordo com os achados de Hasegawa et al. (2015), que sugerem um menor número de sintomas de autismo nos filhos se associou com mais nível de empatia nos pais. A baixa expressão do traço Afago, que representa a busca de apoio e proteção, com sentimentos de abandono e ansiedade, pode indicar uma resiliência dessas mães diante das demandas, mas também a dificuldade de busca de apoio em outras pessoas, refletindo um traço a pouca sociabilidade presente em pessoas com TEA (Yucel et al., 2015).

A Afiliação diz respeito à ligação com pessoas amigas, que pode apresentar percentuais baixos tanto pela maior demanda de cuidados com o filho com TEA e/ou reflexo da exclusão social que a doença do filho pode acarretar para a família (Pondé \& Rousseau, 2013), como fazer parte de um traço do espectro do autismo (Yucel et al., 2015). A prevalência maior de escores baixos para o traço Agressão, por seu turno, pode ter relação com uma posição de retraimento frente à fragilidade diante da situação de vulnerabilidade do filho (Pondé \& Rousseau, 2013). Os baixos escores no traço de Mudança também podem refletir a tendência a rigidez característica do espectro do autismo (Billeci et al., 2016).

Esse estudo tem como limitações importantes o pequeno tamanho da amostra e a ausência de uma população de referencia com crianças com desenvolvimento típico e crianças com outros transtornos do neurodesenvolvimento, como Síndrome de Down. Além disso, não leva em conta os traços de personalidade dos pais, informação que certamente poderia ampliar os dados aqui descritos. Apesar das limitações explanadas, este estudo preliminar pode servir como indicador para pesquisas futuras 
com populações maiores que possam explorar, de forma mais confiável, a associação entre traços de personalidade das mães e gravidade dos sintomas do espectro do autismo nos filhos com diagnóstico de TEA.

\section{CONSIDERAÇÕES FINAIS}

Apesar na natureza exploratória deste estudo, podemos especular que a baixa expressão de alguns traços nas mães de crianças com TEA pode indicar a presença de traços semelhantes àqueles presentes em pessoas com espectro do autismo, relacionados, assim, a marcadores genéticos comuns ao espectro do autismo; podendo ainda se tratar de traços adquiridos a partir de uma complexa interação entre fatores genéticos e as demandas ambientais.

\section{AGRADECIMENTOS}

À Fundação de Amparo ao Pesquisador do Estado da Bahia - FAPESB pelas bolsas PIBIC (BOL0369/2014)

\section{CONTRIBUIÇÕES DOS AUTORES}

Canone L participou da digitação dos dados e análise dos IFPs, revisão da literatura e redação dos resultados. Ananda Genonádio participou da digitação dos dados e análise dos IFPs. Rocha LAS coletou os dados com os pais (questionários, digitação dos dados $\mathrm{e}$ gráficos do artigo). Merelles $\mathrm{S}$ coletou os dados de avaliação das crianças. Coelho MTAD fez a orientação na aplicação e análise dos IFPs. Pondé MP coordenou a coleta de dados, avaliação de crianças, treinamento da equipe, supervisão da equipe e redação do artigo.

\section{CONFLITOS DE INTERESSES}

Nenhum conflito financeiro, legal ou político envolvendo terceiros (governo, empresas e fundações privadas, etc.) foi declarado para nenhum aspecto do trabalho submetido (incluindo mas não limitandose a subvenções e financiamentos, conselho consultivo, desenho de estudo, preparação de manuscrito, análise estatística, etc).

\section{REFERÊNCIAS}

American Psychiatric Association (APA). (2014). Manual diagnóstico e estatístico de transtornos mentais: DSM-5. Artmed Editora.

Billeci, L., Calderoni, S., Conti, E., Gesi, C., Carmassi, C., Dell'Osso, L., ... \& Guzzetta, A. (2016). The broad autism (Endo) phenotype: neurostructural and neurofunctional correlates in parents of individuals with autism spectrum disorders. Frontiers in Neuroscience, Jul 22; 10:346. doi: $10.3389 /$ fnins. 2016.00346

Cook, E. H., Charak, D. A., Arida, J., Spohn, J. A., Roizen, N. J., \& Leventhal, B. L. (1994). Depressive and obsessivecompulsive symptoms in hyperserotonemic parents of children with autistic disorder. Psychiatry research, 52(1), 25-33.

Hasegawa, C., Kikuchi, M., Yoshimura, Y., Hiraishi, H., Munesue, T., Takesaki, N., \& Asada, M. (2015). Changes in autistic trait indicators in parents and their children with ASD: a preliminary longitudinal study. Psychiatry research, 228(3), 956-957. doi: 10.1016/i.psychres.2015.05.048

Machado, S. B., Jr., Celestino, M. I. O., Serra, J. P. C., Caron, J., \& Pondé, M. P. (2016). Risk and protective factors for symptoms of anxiety and depression in parents of children with autism spectrum disorder. Developmental neurorehabilitation, 9(3), 146-153. doi: 10.3109/17518423.2014.925519

Millon, T., \& Roger, O. D. (1996). Disorders of personality: DSMIV and beyond. John Wiley \& Sons.

Mouridsen, S. E., Rich, B., Isager, T., \& Nedergaard, N. J. (2007). Psychiatric disorders in the parents of individuals with infantile autism: a case-control study. Psychopathology, $40(3), 166-171$.

Murphy, M., Bolton, P. F., Pickles, A., Fombonne, E., Piven, J., \& Rutter, M. (2000). Personality traits of the relatives of autistic probands. Psychological medicine, 30(6), 1411 1424.

Novaes, M. C, Pondé, M. P., \& Freire, A. C. C. (2008). Control of psychomotor agitation and agressive behavior in patients with autistic disorder: a retrospective chart review. Arq Neuropsiquiatr, 66(3-B), 646-651. doi: 10.1590/S0004$\underline{282 \times 2008000500008}$

Pasquali, L., Azevedo, M. M., \& Ghesti, I. (1997). Inventário fatorial de personalidade: manual técnico e de avaliação. São Paulo: Casa do Psicólogo.

Piven, J., Palmer, P., Landa, R., Santangelo, S., Jacobi, D., \& Childress, D. (1997). Personality and language characteristics in parents from multiple-incidence autism families. American journal of medical genetics, 74(4), 398-411.

Pondé M. P., Novaes, C. M., \& Losapio, M. F. (2010). Frequency 
of symptoms of attention deficit and hyperactivity disorder in autistic children. Arq neoropsiquiatr, 68(1), 103-106. doi: $10.1590 /$ S0004-282X2010000100022

Pondé, M. P., \& Rousseau, C. (2013). Immigrant children with autism spectrum disorder: the relationship between the perspective of the professionals and the parents' point of view. Journal of the Canadian Academy of Child and Adolescent Psychiatry, 22(2), 131-8.

Rocha, L. A. S. (2015). Perfil de personalidade de pais de crianças com autismo. 2015. Trabalho de conclusão de curso não-publicado, Escola Bahiana de Medicina e Saúde Pública, Salvador, Bahia, Brasil.

Shu, B. C., Lung, F. W., \& Chang, Y. Y. (2000). The mental health in mothers with autistic children: a case-control study in southern Taiwan. The Kaohsiung journal of medical sciences, 16(6), 308-314.

Yucel, G. H., Belger, A., Bizzell, J., Parlier, M., Adolphs, R., \& Piven, J. (2015). Abnormal neural activation to faces in the parents of children with autism. Cerebral Cortex, 25(12), 4643-66. doi: $10.1093 /$ cercor/bhul 47 